\title{
Generador de Agua mediante el uso de Celdas Peltier y Energía Solar
}

\section{Water Generator through Peltier Cells and Solar Energy}

\author{
CHÁVEZ, José Manuel †**, ESPEJEL-BLANCO, Daniel Fernando, HERNÁNDEZ-AGUIRRE, Fredy \\ Alberto y RASCÓN-BARCELÓ, Luis Eduardo
}

Instituto Tecnológico De Hermosillo / Tecnológico Nacional de México

ID 1 ${ }^{\text {er }}$ Autor: José Manuel, Chávez / ORC ID: 0000-0001-9708-5925, CVU CONACYT ID: 806766

ID $1^{\mathrm{er}}$ Coautor: Daniel Fernando, Espejel-Blanco / ORC ID: 0000-0002-9692-013X, CVU CONACYT ID: 304845

ID $2^{\text {do }}$ Coautor: Fredy Alberto, Hernández-Aguirre / ORC ID: 0000-0001-9208-5299, CVU CONACYT ID: 611800

ID $3^{\text {er }}$ Coautor: Luis Eduardo, Rascón-Barceló / ORC ID: 0000-0001-6051-9777, CVU CONACYT ID: 929775

DOI: $10.35429 /$ JOIE.2019.11.3.22.28

Recibido 13 de Junio, 2019; Aceptado 30 de Septiembre, 2019

\section{Resumen}

En la actualidad, las épocas de sequía son más prolongadas a consecuencia del calentamiento global, lo cual ha generado escases y racionamiento de agua, dichas condiciones se convierten en el principal problema en los poblados alejados a las zonas urbanas y con poca infraestructura. México cuanta con 653 acuíferos en todo su territorio, 106 están sobreexplotados debido a actividades agrícolas, mineras, industriales y urbanas, lo que proyecta al país un posible agotamiento y contaminación de su líquido vital. Por tal motivo, con el fin de contribuir con una solución a la problemática de la escases de agua, al aplicar un sistema de control a la tecnología que utilizan las celdas de Peltier se genera un flujo moderado de agua, ya que condensa las partículas de agua suspendidas en el aire. De esta manera se implementara un arreglo de celdas de Peltier para obtener la mayor cantidad agua posible bajo factores ambientales, como humedad y temperatura. Además, al tratarse de zonas donde el clima es árido, se pretende utilizar fuentes de energía alternativos, de tipo fotovoltaico autónomo, para aprovechar la radiación solar y alimentar al sistema de control de una manera autosustentable.

Energías Renovables, Condensación de Agua, Celdas de Peltier

\begin{abstract}
At present, the drought periods are longer due to global warming, which has generated scarcity and water rationing, these conditions become the main problem in remote villages to urban areas and with little infrastructure. Mexico, with 653 aquifers throughout its territory, 106 are overexploited due to agricultural, mining, industrial and urban activities, which projects to the country a possible depletion and contamination of its vital liquid. For this reason, in order to contribute to a solution to the problem of water scarcity, by applying a control system to the technology used by the Peltier cells, a moderate flow of water is generated, since it condenses the particles of water suspended in the air. In this way, an array of Peltier cells will be implemented to obtain as much water as possible under environmental factors, such as humidity and temperature. In addition, when dealing with areas where the climate is arid, it is intended to use alternative sources of energy, of autonomous photovoltaic type, to take advantage of solar radiation and feed the control system in a self-sustaining manner.
\end{abstract}

Renewable Energies, Water Condensation, Peltier Cells

Citación: CHÁVEZ, José Manuel, ESPEJEL-BLANCO, Daniel Fernando, HERNÁNDEZ-AGUIRRE, Fredy Alberto y RASCÓN-BARCELÓ, Luis Eduardo. Generador de Agua mediante el uso de Celdas Peltier y Energía Solar. Revista de Ingeniería Innovativa. 2019. 3-11: 22-28

\footnotetext{
*Correspondencia al Autor (Correo electrónico: jmch.ith@gmail.com)

$\dagger$ Investigador contribuyendo como primer Autor.
} 


\section{Introducción}

En la actualidad, el hablar sobre el cambio climático es referirse al principal problema que nuestro planeta enfrenta y el cual, no solo afecta a nuestros distintos ecosistemas, sino también a la sociedad. Las temperaturas del planeta son aptas para la vida gracias a un proceso natural llamado "Efecto Invernadero"; cuando la radiación solar llega a nuestra atmosfera, parte de ella es reflejada hacia el espacio y otra parte es absorbida por la Tierra, obteniendo la temperatura adecuada para la vida en ella.

Por otra parte, existen los llamados "Gases de Efecto Invernadero", los cuales evitan que la radiación solar salga hacia el espacio y se quede en nuestra atmosfera, ocasionando un incremento en las temperaturas.

México no es la excepción, a consecuencia del calentamiento global el país experimenta distintos problemas climáticos, los cuales nunca se habían registrado. El incremento de temperatura cada vez es más prolongado en épocas de sequía, donde el suelo es árido, se ha generado escases y racionamiento de agua, dichas condiciones se convierten en la mayor afectación en los pueblos alejados a las zonas urbanas y con poca infraestructura.

El agua con el que se cuenta, muchas veces por condiciones naturales, y otras por la intervención del ser humano, llega a sufrir daños irreversibles. México cuenta con 653 acuíferos en todo su territorio, 106 están sobreexplotados debido a actividades agrícolas, mineras, industriales y urbanas, lo que proyecta al país un posible agotamiento y contaminación de su líquido vital (Comisión Nacional del Agua, 2018).

Al tratarse de zonas donde el clima es árido, el acceso a fuentes ilimitadas de este líquido vital es imposible, afectando al desarrollo de empresas y comunidades. Se han realizado múltiples investigaciones sobre los métodos alternativos para la obtención de agua, logrando resultados no viables. Dado a los altos costos de transportación de agua potable entre distintas localidades y a la sobreexplotación de los mantos acuíferos pareciera que nuestro problema no tendrá fin.
Ante el evidente problema que nuestro planeta enfrenta a consecuencia del cambio climático y el racionamiento del agua, como ingenieros y futuros investigadores es nuestra obligación desarrollar y aplicar nuevas tecnologías para aprovechar los recursos del planeta, como es el caso de la implementación de sistemas fotovoltaicos.

El aprovechamiento de la energía solar para generar electricidad a través de la tecnología fotovoltaica se ha convertido en una opción cada vez más asequible para los 1400 millones de personas en el mundo que viven aún sin energía eléctrica (Rodriguez \& Espinoza, 2017). Dependiendo de las necesidades humanas, el sistema fotovoltaico puede variar en tipo, capacidad o especificaciones. Así, los tipos de sistemas fotovoltaicos se dividen en dos principales: los autónomos o aislados y los interactivos con la red eléctrica o interconectados.

El funcionamiento de un sistema fotovoltaico es posible gracias al a los paneles solares donde gracias al efecto fotoeléctrico, la energía solar se convierte en energía eléctrica de corriente directa, el cual puede ser convertido a corriente altera al utilizar el inversor, esto dependerá de los dispositivos electrónicos que se deseen alimentar con el sistema fotovoltaico.

Posteriormente, dependiendo del tipo de sistema fotovoltaico: autónomo o interconectado, en el caso de sistemas fotovoltaicos autónomos es necesario utilizar controladores de cargas que regulan el uso de la energía y un banco de baterías que permita el almacenamiento de la energía.

Por otra parte, el uso de centros de carga es fundamental en sistemas fotovoltaicos interconectados, ya que es el punto de conexión o de distribución de energía fotovoltaica generada para que el medidor bidireccional registre la energía que se regresa a la red eléctrica de CFE (Rodriguez \& Espinoza, 2017). Por tal motivo, es necesario un sistema capaz de generar agua de manera alterna, el cual, no afecte a nuestro ecosistema y pueda satisfacernos de un flujo moderado de agua. 
El objetivo de este proyecto es la es la implementación de un prototipo generador de agua utilizando Celdas Peltier, basadas en el principio termoeléctrico Peltier, para condensar el vapor de agua contenido en el aire para obtener rocío. El punto de rocío o temperatura de rocío es la más alta temperatura a la que empieza a condensarse el vapor de agua contenido en el aire, produciendo rocío, neblina, cualquier tipo de nube o, en caso de que la temperatura sea lo suficientemente baja, escarcha (MetAs \& Metrologos Asociados, 2007).

Con este sistema es posible obtener la mayor cantidad de agua posible bajo factores ambientales, como humedad y temperatura, en relación a la manipulación y control de las Celdas de Peltier. A su vez, utilizar una fuente de energía alternativa autónoma, de tipo fotovoltaica, para la alimentación del sistema en zonas donde no exista acceso a fuentes de energía eléctrica constante.

\section{Desarrollo}

El prototipo está integrado de tal manera de generar la máxima cantidad de agua bajo distintos factores ambientales. Para el desarrollo del prototipo se utilizaron diversos fundamentos teóricos, principalmente Termoelectricidad, así como los principios del Efecto Peltier.

Una forma de generar la máxima cantidad de agua utilizando la humedad del ambiente es con la implementación de Celdas de Peltier, la cual si se le aplica una corriente eléctrica, en un circuito compuesto de materiales distintos con sus uniones, absorbe el calor de una de ellas y se desprende por la otra, es decir, se genera una diferencia de temperatura entre sus caras al ser circuladas por una corriente: una de ellas se enfría, mientras que la otra se calienta (Tornos \& Sotelo, 2006).

Si se invierte la polaridad de la corriente, la cara fría pasa a ser caliente y viceversa, invirtiéndose el sentido del flujo calorífico, llevando por nombre Efecto Peltier (Patterson \& Sobral, 2007).

$\mathrm{Al}$ entrar en contacto la parte fría de la Celda Peltier con al aire del ambiente, la cual se encuentra a cierta temperatura y con un porcentaje de humedad, comienza a condensar el vapor de agua contenido en el aire, produciendo rocío.
Es decir, es la temperatura a la cual el vapor de agua presente, alcanza su valor máximo o su condición de presión de saturación de vapor de agua con respecto al equilibrio con una superficie plana de agua. La temperatura de punto de rocío es siempre menor que o igual a la temperatura actual.

El prototipo está complementado con sensores para la medición de temperatura y humedad, las cuales determinaran las condiciones de activación del prototipo utilizando un control ON/OFF mediante la plataforma de Arduino. El cual es una plataforma de creación de electrónica de código abierto, la cual está basada en hardware y software libre, flexible y fácil de utilizar para los creadores y desarrolladores, la cual crear diferentes tipos de microordenadores de una sola placa a los que la comunidad de creadores puede darles diferentes tipos de uso (Electronics, s.f.).

La estructura principal del prototipo consiste básicamente en tres etapas: adquisición de información, sistema de control y fuente de alimentación, siendo las dos primeras interconectadas a través de una arquitectura desarrollada a partir de la plataforma Arduino.

\section{Arquitectura de Arduino para recolección de datos}

Utilizando la plataforma de programación Arduino, como se muestra en la Figura 1, en conjunto con los sensores de humedad y temperatura se determinan los parámetros de activación de las Celdas Peltier para generar el punto de rocío.

La placa Mega 2560, mostrado en la Figura 2, gracias a su interacción con distintos tipos de sensores, así como sus puertos de entradas y salidas digitales, se implementó en el sistema para realizar el muestreo de los datos ambientales. La Tabla 1 muestra la lista de materiales utilizados en el muestreo de variables. 


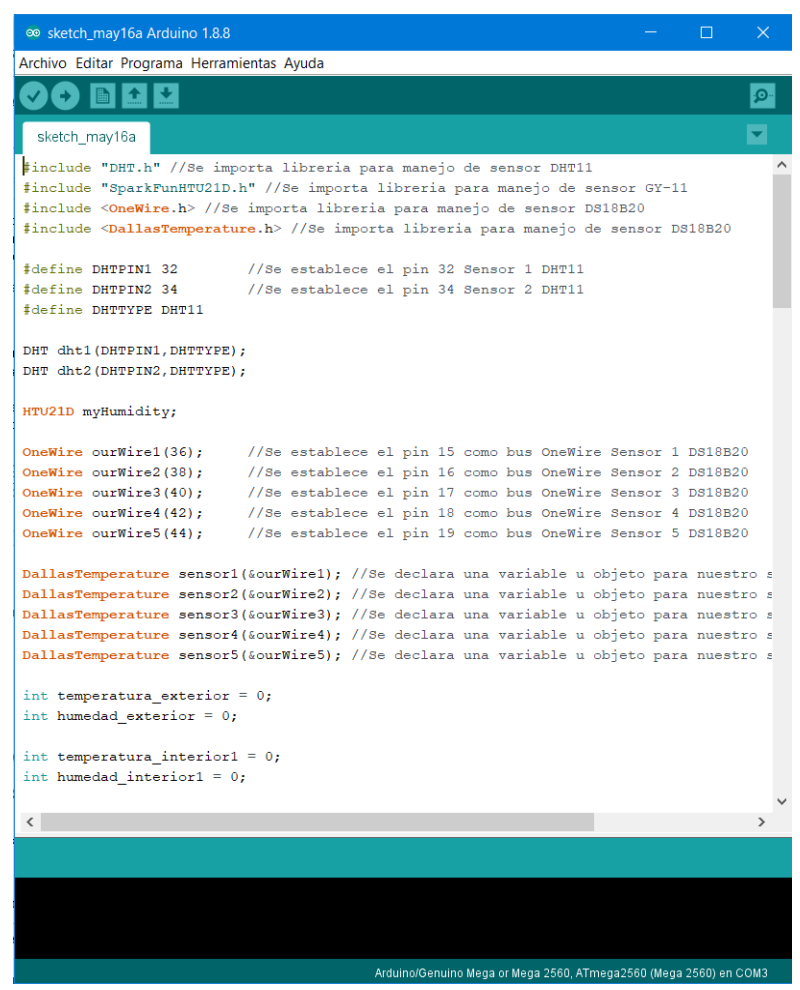

Figura 1 Programa en Arduino para el control ON/OFF del prototipo

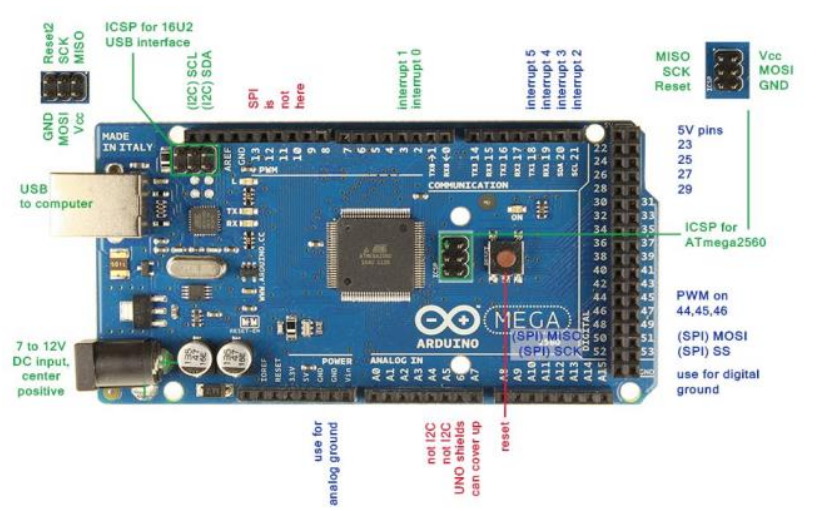

Figura 2 Placa Arduino Mega 2560

$\mathrm{Al}$ estar monitoreando el sistema en tiempo real, es posible determinar bajo qué condiciones el sistema generador de agua presenta mejores resultados como se muestran en la figura 3 .

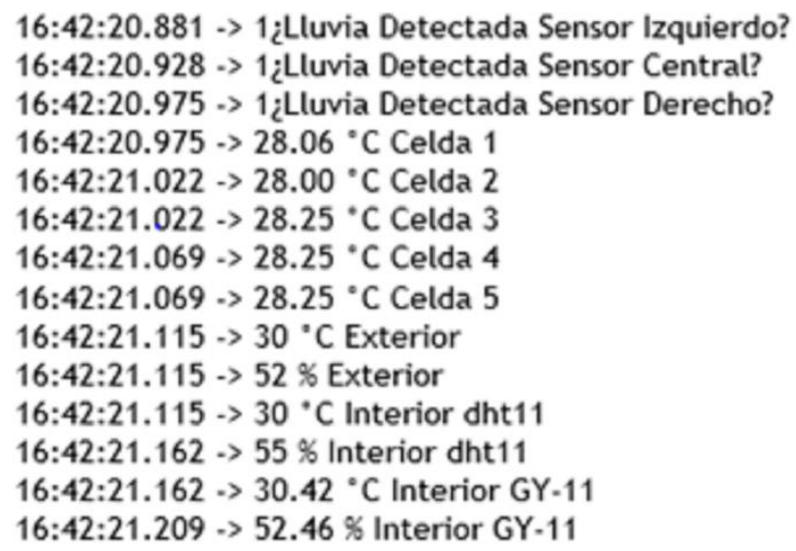

Figura 3 Datos obtenidos a través de sensores y procesados en Plataforma Arduino

\begin{tabular}{|l|ll|l|}
\hline Cantidad & \multicolumn{1}{c}{ Descripción } & \multicolumn{1}{c|}{ Parte } \\
\hline 5 & Sensor de Temperatura & DS18B20 \\
\hline 2 & $\begin{array}{l}\text { Sensor de Temperatura y } \\
\text { Humedad }\end{array}$ & DHT11 \\
\hline 1 & $\begin{array}{l}\text { Sensor de Temperatura y } \\
\text { Humedad }\end{array}$ & GY-21 \\
\hline
\end{tabular}

Tabla 1 Lista de materiales para medir humedad relativa y temperatura

\section{Arquitectura del Actuador de Celdas Peltier}

Al utilizar la tarjeta Mega 2560 y su plataforma Arduino, el sistema es puesto en funcionamiento en base a las variables medidas, humedad relativa y temperatura, con la finalidad de entrar en operación cuando las variables mencionadas sean óptimas para la mayor obtención de rocío. La Figura 4 muestra la relación entre temperatura ambiente y porcentaje de humedad relativa para obtener la temperatura a la que se presentara el punto de rocío.

Si los factores ambientales son ideales, la tarjeta Mega 2560 envía una señal por medio de su salida digital al módulo del actuador, como se muestra en la Figura 5, que a su vez alimentara a la Celda Peltier, como puede observarse en la Figura 6.

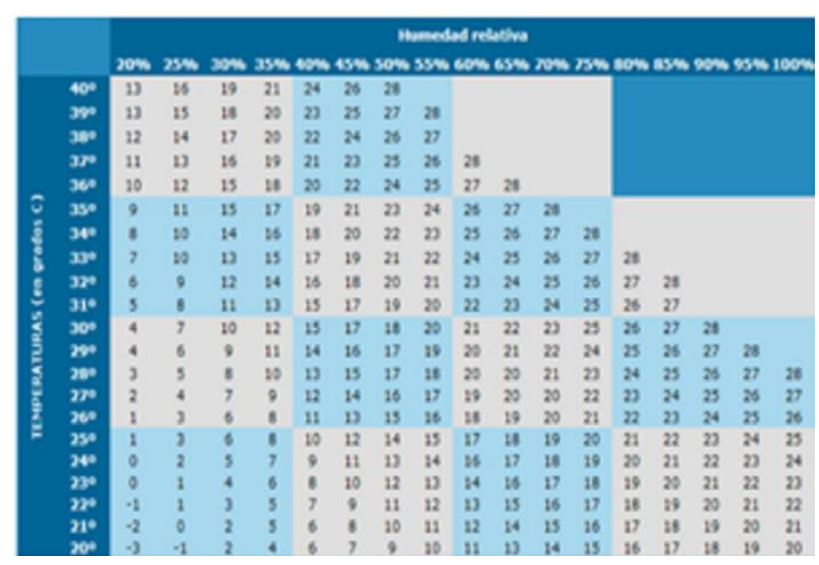

Figura 4 Relación entre temperatura, humedad relativa y punto de rocío

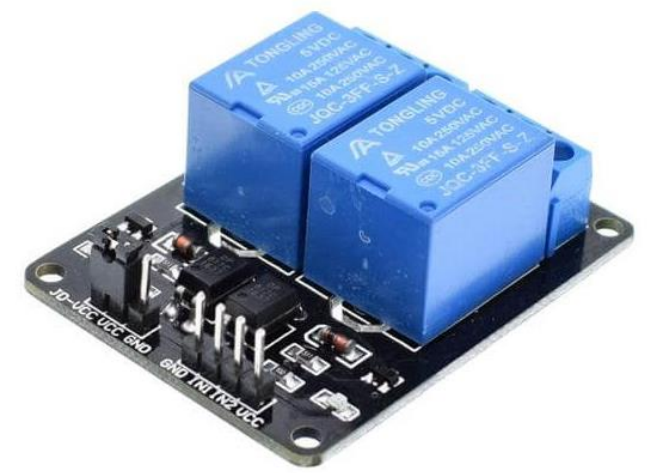

Figura 5 Modulo Actuador-Relevador

CHÁVEZ, José Manuel, ESPEJEL-BLANCO, Daniel Fernando, HERNÁNDEZ-AGUIRRE, Fredy Alberto y RASCÓN-BARCELÓ, Luis Eduardo. Generador de Agua mediante el uso de Celdas Peltier y Energía Solar. Revista de Ingeniería Innovativa. 2019 


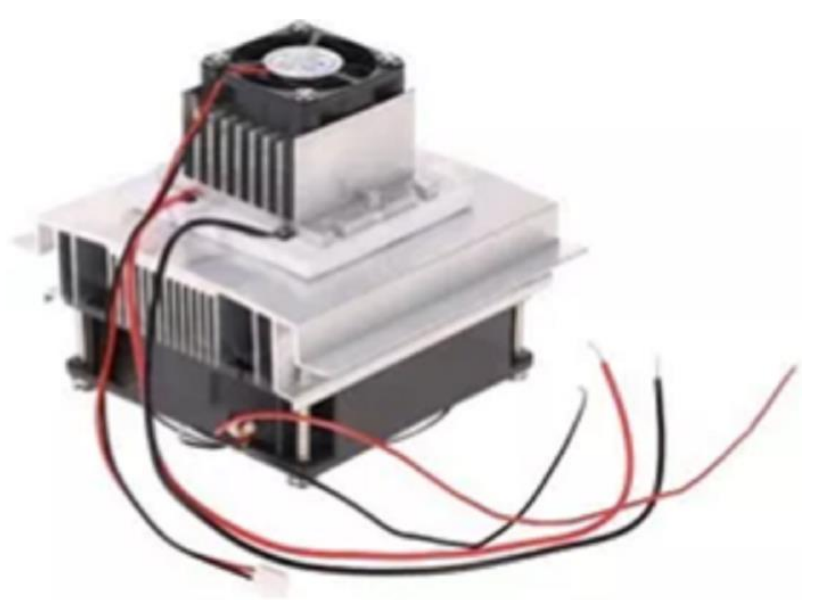

Figura 6 Kit Celda Peltier con sistema de enfriamiento

Al activar las Celdas Peltier, estas comienzan a disminuir su temperatura hasta alcanzar $0^{\circ} \mathrm{C}$, si al entrar en contacto con una mezcla de humedad y temperatura se enfría a una presión constante, sin variar el contenido de vapor de agua que tiene el aire, se llega a una temperatura en la que el vapor comienza a condensar. Esa temperatura es la llamada punto de rocío y a esa temperatura la humedad relativa será del $100 \%$.

\section{Conexión del Sistema Fotovoltaicos Autónomo como fuente de alimentación}

En la Figura 7 se muestra la estructura del Sistema Fotovoltaico Autónomo utilizado como fuente de alimentación en el prototipo generador de agua. La electricidad en corriente continua es la que proviene de los módulos fotovoltaico y que se almacena en las baterías. Si el prototipo es puesto en operación durante el día utilizara directamente la energía generada por los módulos fotovoltaicos, contrario a esto, en ausencia de luz solar el sistema se alimentara de los acumuladores, que previamente almacenaron energía eléctrica durante el día.

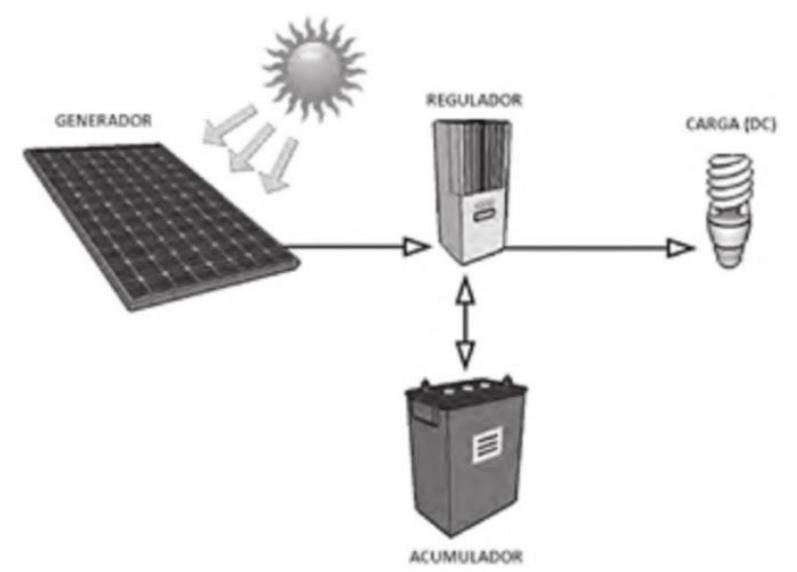

Figura 7 Sistema Fotovoltaico Autónomo con salida en CD

\section{Resultados}

Los resultados obtenidos demuestran el desempeño de las tecnologías para resolver las necesidades básicas de la sociedad. Al diseñar nuevos métodos o sistemas para la obtención de agua, podemos aprovechar los distintos ecosistemas y estaciones del año para generar la máxima cantidad posible, de una manera amigable para el medio ambiente y a un bajo costo.

La cantidad de agua generada será proporcional al número de Celdas Peltier utilizadas, la humedad presente en el ambiente y el tiempo de operación del sistema, por ejemplo, en un tiempo estimado de seis horas la máxima cantidad de agua producida fue de $300 \mathrm{~mL}$ con el $70 \%$ de humedad. En la Figura 8 se presenta el prototipo operando con la implementación de cinco Celdas Peltier y ventiladores por la parte lateral para la circulación de aire y en la Figura 9 el circuito de control.

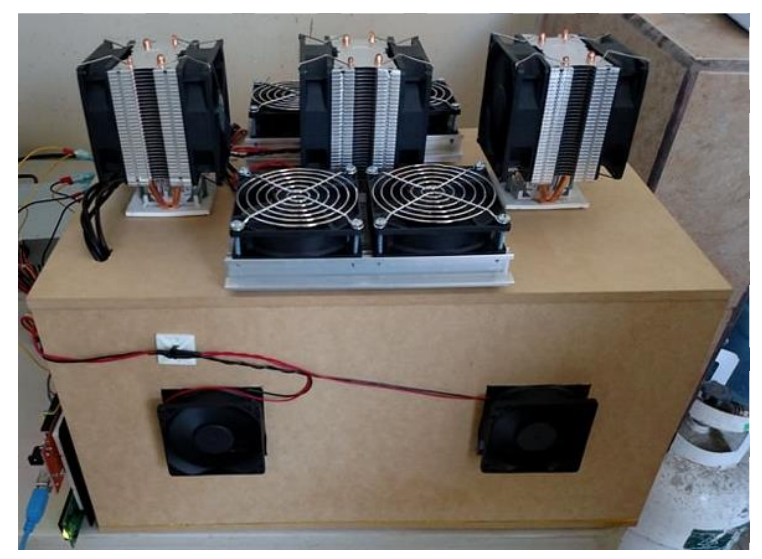

Figura 8 Prototipo Generador de Agua

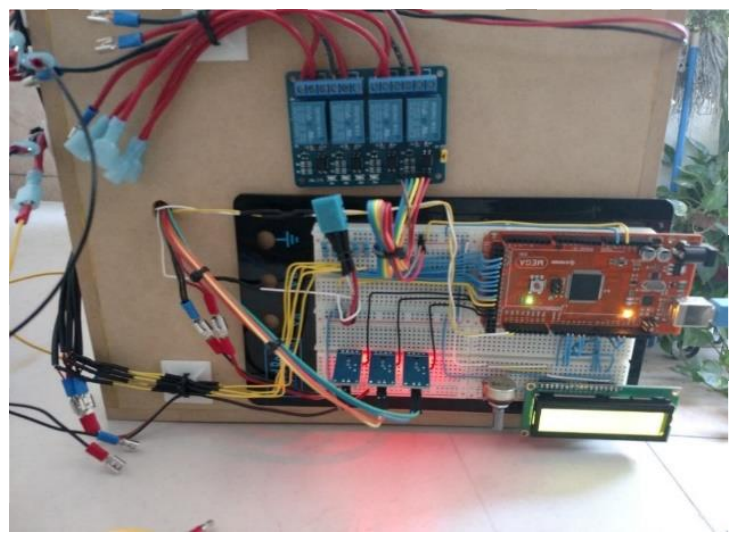

Figura 9 Sistema de Control conectado a sensores y a actuadores
CHÁVEZ, José Manuel, ESPEJEL-BLANCO, Daniel Fernando, HERNÁNDEZ-AGUIRRE, Fredy Alberto y RASCÓN-BARCELÓ, Luis Eduardo. Generador de Agua mediante el uso de Celdas Peltier y Energía Solar. Revista de Ingeniería Innovativa. 2019 


\section{Agradecimientos}

Agradecimiento especial al Tecnológico Nacional de México/Instituto Tecnológico de Hermosillo, por proporcionar los fondos para que este proyecto se llevara a cabo.

\section{Conclusiones}

El prototipo propuesto para la generación de agua es una excelente opción en zonas donde no exista su acceso, ya que no requiere un alto costo de implementación. Los resultados obtenidos con las pruebas realizadas, nos brinda las bases necesarias para implementar un sistema generador y adaptarlo en sitios con alto porcentaje de humedad. Ya que el sistema de control funciona bajo los mismos criterios, solo es necesario dimensionar el prototipo dependiendo de la cantidad de agua que generara y sus aplicaciones. Por otra parte, puede ser implementado en zonas donde no exista suministro constante de energía eléctrica, gracias a su fuente de alimentación autosustentable.

\section{Recomendaciones}

El prototipo propuesto sugiere algunas pruebas y modificaciones tanto a nivel hardware como software. Se propone la utilización de la plataforma WIFI, en reemplazo a la plataforma Arduino, para el monitoreo y control del prototipo generador. Con lo cual se tendrá un mejor manejo de datos a distancia interconectados a la red local y a su vez, una disminución de dimensionamiento de circuitería.

\section{Referencias}

Aguirre, J. (2019). Autobiogen: automatización de un biodigestor de cinco propósitos. Aplicación al proceso de climatización, combustible para una estufa doméstica, producción de energía eléctrica, reciclaje del agua residual y generación de fertilizante en una casa habitación para cuatro personas (Tesis doctoral) Universidad Autónoma Metropolitana. México, D. F., México.

Comisión Nacional del Agua. (2018). Situación de los Recursos Hídricos. Recuperado desde https://www.gob.mx/conagua/acciones-yprogramas/situacion-de-los-recursos-hidricos Electronics, M. (2015). Arduino. (2019) Recuperado desde http://arduino.cl/que-esarduino/
Eole Water Company. (2012). Eole Water Company. Recuperado desde: http://www.eolewater.com/

Gómez, J. (2019). Fortalecimiento del sistema de tecnologías apropiadas para la generación de energías limpias en el Parque Natural Chicaque (Tesis de Pregrado). Universidad Distrital Francisco José de Caldas. Bogotá, Colombia.

Martines L., E. L. (2008). Cálculo de la Temperatura de Punto de Rocío a Diferentes Valores de Presión. Simposio de Metrología. SM2008-M117-1098-5 Recuperado desde https://www.cenam.mx/simposio2008/sm_2008 /memorias/M1/SM2008-M117-1098.pdf

MetAs \& Metrologos Asociados. (2007). Temperatura de Punto de Rocío. MetAs \& Metrologos Asociados. 2007-11 Recuperado desde http://www.metas.com.mx/guiametas/LaGuia-MetAs-07-11-punto-de-rocio.pdf

Patterson, G., \& Sobral, M. (2007). Efecto Peltier. FCEyN, UBA. Recuperado desde http://materias.df.uba.ar/labo4aa2014c1/files/20 12/07/Efecto-Peltier.pdf

Planet's Water Company. (2018). Planet's Water Company. Recuperado desde: http://www.waterplanet.com/

Portillo, A. L. (2017). Energía solar en México: su potencial y aprovechamiento. Recuperado desde ciep.mx.

Reina, W. \& Acosta, J. (2019) Sistema de supervisión y control del prototipo generador de agua a partir de celdas de Peltier (Tesis de Pregrado). Universidad Distrital Francisco José de Caldas. Bogotá, Colombia.

Rodríguez, J., \& Espinoza, E. (2017). La Industria Solar Fotovoltaica y Foto térmica en México. ProMéxico, 15-75. Recuperado desde https://www.gob.mx/cms/uploads/attachment/fi le/428621/La_industria_solar_fv_y_ft_en_M_x ico-compressed.pdf

Rojas, A. \& Burgos, E. (2019) Evaluación de alternativas sobre dispositivos de refrigeración con fuentes de energía no convencional para las zonas no interconectadas de Colombia (Tesis de Pregrado). Universidad Distrital Francisco José de Caldas. Bogotá, Colombia. 
Sacristán, A. (2011). Obtención de agua a partir de humedad atmosférica empleando células de efecto Peltier (Tesis de Pregrado). Universidad Carlos III de Madrid. Madrid, España

Sampedro, O. F. B., Pérez, D. F. M., \& Núñez, E. F. V. (2019). Proyecto de transformación del calor residual de gases de combustión de un automóvil en energía eléctrica, como contribución al cuidado del medio ambiente. Ciencia Digital, 3(3.1), 54-73.

sde.mx. (2018). ¿Qué es un sistema fotovoltaico? Recuperado desde http://www.sde.mx/2018/08/10/que-es-unsistema-fotovoltaico/

Style, O. (2012). Energía Solar Autónoma: Planificación, Dimensionado e Instalación de un Sistema Fotovoltaico Autónomo. U.S.A. Itaca Appropriate Technology.

Tornos, S., \& Sotelo, A. (2006). Termoelectricidad, la energía del desequilibrio. Técnica Industrial 262. Recuperado desde http://www.tecnicaindustrial.es/tiadmin/numero s/22/41/a41.pdf

ZeroMass Water. (2018). ZeroMass Water. Recuperado desde https://www.zeromasswater.com/ 